\title{
Surgically induced astigmatism following trabeculectomy
}

\author{
Gyu Ah Kim ${ }^{1} \cdot$ Si Hyung Lee ${ }^{2}$ Sang Yeop Lee ${ }^{1}$ Hee Jung Kwon ${ }^{3} \cdot$ Hyoung Won Bae $^{1} \cdot$ Gong Je Seong ${ }^{1}$. \\ Chan Yun Kim ${ }^{1}$
}

Received: 9 August 2017 / Revised: 25 January 2018 / Accepted: 30 January 2018 / Published online: 14 March 2018

(c) The Royal College of Ophthalmologists 2018

\begin{abstract}
Purpose Surgically induced astigmatism (SIA) has attracted much interest in recent times because changes in corneal astigmatism can lead to decreased uncorrected visual acuity and patient discomfort. This study aimed to evaluate SIA and to identify factors correlated therewith after trabeculectomy.

Methods We retrospectively reviewed medical charts of patients who were treated with trabeculectomy at $120^{\circ}$ meridian (superotemporal area on right eye and superonasal area on left eye) by the same surgeon. Preoperative keratometric data were compared with data collected from 2 months to 12 months postoperatively. SIA was evaluated using Naeser's polar value analysis.

Results Using Naeser's method, $\Delta \mathrm{KP}(120)$ was calculated as $0.7 \pm 0.7\left(0.82 @ 104^{\circ}\right)$, which indicates a with-the-rule change. After surgery, the combined mean polar values changed significantly (Hotelling $T^{2}=22.47 ; p<0.001$ ). Multivariate analysis of variance indicated that postoperative intraocular pressure and location of surgery were independent factors that were significantly associated with SIA ( $p=0.002$ and 0.03 , respectively).

Conclusions Trabeculectomy at the $120^{\circ}$ meridian was not astigmatically neutral. In addition, the SIA after trabeculectomy appears to be greater in eyes with low postoperative intraocular pressure and a superonasal surgical wound rather than a superotemporal wound.
\end{abstract}

\section{Introduction}

Trabeculectomy remains one of the primary surgical options for achieving target intraocular pressure (IOP) in patients with glaucoma. As surgical techniques have developed markedly over the past few decades, the goal of the trabeculectomy has expanded to include not only adequate control of IOP, but successful visual rehabilitation of patients. However, clinicians occasionally encounter cases in which surgically induced corneal changes are more substantial than expected and that impair visual outcomes

$\triangle$ Chan Yun Kim

kcyeye@yuhs.ac

1 Department of Ophthalmology, Institute of Vision Research, Severance Hospital, Yonsei University College of Medicine, Seoul, Korea

2 Department of Ophthalmology, Bucheon Hospital, Soonchunhyang University, Bucheon, Korea

3 Department of Ophthalmology, CHA Bundang Medical Center, CHA University, Seongnam, Korea and patient satisfaction. Therefore, identification of factors that may influence corneal astigmatic change after trabeculectomy is garnering increased attention.

Additionally, surgically induced astigmatism (SIA) after cataract surgery has been extensively investigated. It is well recognized that factors affecting SIA include the location [1, 2], width of the incision [3], and corneal biomedical properties, such as corneal hysteresis [4]. The amount of SIA was shown to range from 0.09 to 1.92 diopters, according to different incisions used for cataract surgery $[2,5,6]$. In contrast, there have been few reports on SIA after trabeculectomy. Thus, the current study retrospectively evaluated SIA after trabeculectomy and the related factors, using vector analysis.

\section{Methods}

\section{Study population}

The research adhered to the tenets of the Declaration of Helsinki, and the study was approved by the Institutional Review Board of Severance Hospital. 
Table 1 Patient demographics

\begin{tabular}{ll}
\hline All patients $(N=51)$ & \\
\hline Age (years) & $48.0 \pm 17.2$ \\
Male sex $(\%)$ & $32(62.7 \%)$ \\
Right eye $(\%)$ & $25(49.0 \%)$ \\
Preoperative IOP (mmHg) & $22.0(18.0 ; 31.0)$ \\
Postoperative IOP (mmHg) & $10.0(7.5 ; 14.0)$ \\
Follow-up period (months) & $5.0(3.0 ; 6.0)$ \\
Axial length (mm) & $25.1(23.6 ; 26.6)$ \\
Number of scleral suture & $4.0(3.0 ; 5.0)$ \\
Laser suture lysis $(\%)$ & \\
0 & $22(43.1 \%)$ \\
1 & $21(41.2 \%)$ \\
2 & $5(9.8 \%)$ \\
3 & $2(3.9 \%)$ \\
4 & $1(2.0 \%)$ \\
Number of needling $(\%)$ & \\
0 & $43(84.3 \%)$ \\
1 & $4(7.8 \%)$ \\
2 & $2(3.9 \%)$ \\
3 & $1(2.0 \%)$ \\
4 & $1(2.0 \%)$ \\
\hline
\end{tabular}

$I O P$ intraocular pressure

We retrospectively reviewed medical charts of openangle glaucoma patients who were treated with trabeculectomy at the $120^{\circ}$ meridian (the superotemporal area of the right eye and the superonasal area of the left eye) by the same surgeon (C.Y.K) in the Severance hospital (Seoul, Korea), from January 2011 to December 2015. Eyes with previous ocular surgery or less than 2 months of follow-up data were excluded.

All patients underwent comprehensive ophthalmologic examinations, including slit lamp examination, measurements of IOP using Goldmann applanation tonometry, keratometry using an autokeratometer (RK-3, Canon, Lake Success, NY), and axial length using optical biometry (IOL Master, Carl Zeiss Meditec, Jena, Germany). In addition, patient data on demographics and clinical factors (follow-up period of postoperative keratometry measurement, number of sutures on the scleral flap, status of suture lysis or needling) were extracted from medical records. Laser suture lysis was performed within the second postoperative week before keratometry measurement. Based on preoperative and postoperative keratometric data from 2 months to 12 months after surgery, SIA was evaluated using Naeser's polar value analysis [7-9].

\section{Naeser's polar value analysis}

Based on the polar value method [8], a net astigmatism with a magnitude in diopters and direction in degrees is converted to a pair of polar values in diopter units, separated by an $\operatorname{arc}$ of $45^{\circ}(\mathrm{KP}(\Phi), \mathrm{KP}(\Phi+45)) . \mathrm{KP}(\Phi)$ is defined as the difference between the meridional power along $\Phi$ and its orthogonal meridian $(\Phi+90)$, and indicates the net refractive power acting along the plane $\Phi$. Similarly, $\operatorname{KP}(\Phi$ $+45)$ is the difference between the powers of the oblique meridians $(\Phi+45)$ and its orthogonal meridian $(\Phi-45)$ and indicates the power twisting the astigmatic direction toward the plane through either $(\Phi+45)$ or $(\Phi-45)$, in other words, the net torsional power over $\Phi$. In addition, SIA, expressed as polar values $(\Delta \mathrm{KP}(\Phi), \Delta \mathrm{KP}(\Phi+45))$, is the difference between the postoperative and the preoperative polar values. Because the trabeculectomy was performed at the $120^{\circ}$ meridian in this study, we obtained $\mathrm{KP}(120)$ and $\mathrm{KP}(165)$. Herein, a positive $\Delta \mathrm{KP}(120)$ indicates a surgically induced steepening (with-the-wound change), and a negative $\triangle \mathrm{KP}(120)$ indicates a surgically induced flattening of the surgical meridian (against-the-wound change). A positive $\Delta \mathrm{KP}(165)$ represents a counter-clockwise torque, while a negative $\Delta K P(165)$ indicates a clockwise torque.

\section{Surgical techniques of trabeculectomy and postoperative managements}

All patients underwent a partial thickness trabeculectomy, with application of intraoperative mitomycin-C. After a limbal-based conjunctival flap was made, a $4 \times 3$-mm-sized rectangular scleral flap was created at the $120^{\circ}$ meridian (superotemporal area of the right eye and the superonasal area of the left eye). Subsequently, a sponge soaked in $0.04 \%$ mitomycin-C was placed in the subconjunctival space for $3 \mathrm{~min}$. Following vigorous irrigation, using balanced salt solution, of the area exposed to mitomycin-C, trabeculectomy and peripheral iridectomy were performed. The scleral flap was closed with several interrupted 10-0 nylon sutures. After gauging the proper flow through the filter site, the number of sutures was adjusted as necessary. Then, the conjunctival flap was sutured continuously with 7-0 Vicryl (Ethicon Inc., Somerville, NJ). Laser suture lysis or bleb needling were considered in cases with poor postoperative IOP control.

\section{Statistical analysis}

Descriptive statistics for all demographic and clinical variables were calculated. Continuous variables were expressed as mean \pm standard deviation values or median values (interquartile range), whereas categorical variables were expressed as absolute and relative frequencies. To compare the preoperative and the postoperative mean polar values, a univariate analysis was performed with the paired $t$-test and a bivariate analysis was performed with the Hotelling trace test, as described previously [10]. Furthermore, to evaluate 
Table 2 Polar value analysis

\begin{tabular}{|c|c|c|c|c|c|c|c|c|c|}
\hline & \multicolumn{6}{|c|}{ Mean keratometry $(D) \pm \mathrm{SD}$} & \multirow[b]{3}{*}{$p$ Value $^{\mathrm{b}}$} & \multirow{2}{*}{\multicolumn{2}{|c|}{$p$ Value $^{\mathrm{a}}$}} \\
\hline & \multicolumn{2}{|c|}{ Preoperative } & \multicolumn{2}{|c|}{ Postoperative } & \multicolumn{2}{|l|}{ Change } & & & \\
\hline & $\mathrm{KP}(120)$ & $\mathrm{KP}(165)$ & $\mathrm{KP}(120)$ & $\mathrm{KP}(165)$ & $\Delta \mathrm{KP}(120)$ & $\Delta \mathrm{KP}(165)$ & & $\Delta \mathrm{KP}(120)$ & $\Delta \mathrm{KP}(165)$ \\
\hline Total $(N=51)$ & $0.3 \pm 0.6$ & $-0.2 \pm 0.7$ & $1.0 \pm 1.0$ & $-0.6 \pm 1.2$ & $0.7 \pm 0.7$ & $-0.4 \pm 0.9$ & $<0.001$ & $<0.001$ & 0.001 \\
\hline
\end{tabular}

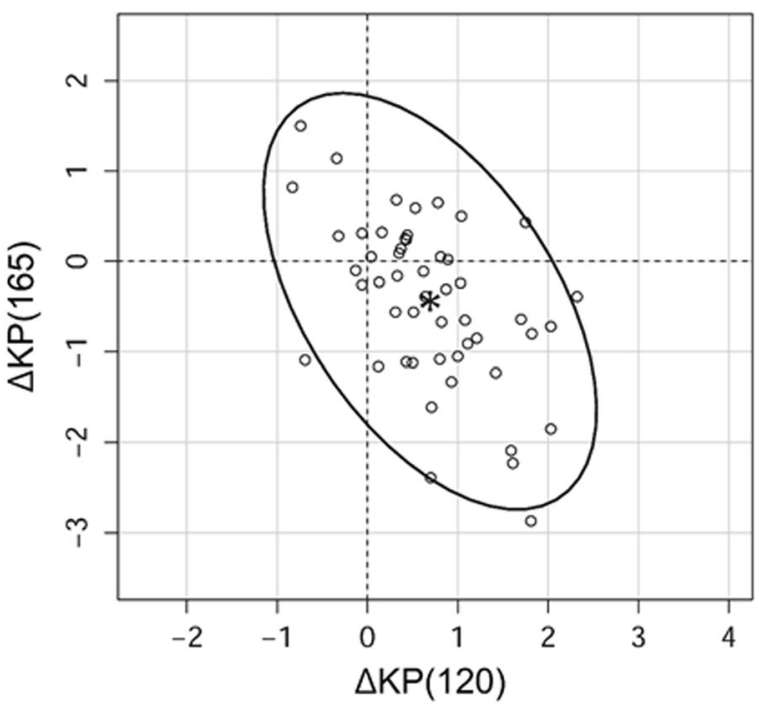

Fig. 1 Bivariate analysis of surgical induced astigmatism. The asterisk denotes the combined mean value of polar values and $95 \%$ bivariate confidence ellipse is inserted. $\Delta=$ change; $\mathrm{KP}=$ astigmatic polar value

the effect of clinical factors on SIA, multivariate analysis of variance (MANOVA) was conducted. The assumptions (independence, normality, and homogeneity of variance of the residuals) for MANOVA were tested and were met. Statistical significance was set at $p<0.05$. All statistical analyses were performed using R software version 3.1.2 (R Foundation, Vienna, Austria).

\section{Results}

Fifty-one eyes of 51 patients were included in the study. Table 1 shows the clinical characteristics of the participants. Their mean age was $48.0 \pm 17.2$ years and the median follow-up period, the time at which postoperative keratometry was performed, was 5 months. Twenty-five patients underwent trabeculectomy at the superotemporal limbus of the right eye, while others underwent the procedure at the superonasal limbus of the left eye. There were 29 patients $(56.9 \%)$ who required laser suture lysis and eight patients $(15.7 \%)$ who needed needling to achieve the target IOP and produce adequate blebs. The median preoperative and postoperative IOPs were $22 \mathrm{mmHg}$ and $10 \mathrm{mmHg}$, respectively.

Table 2 shows the result of corneal polar value analysis. The univariate analysis revealed that $\mathrm{KP}(120)$ increased and $\mathrm{KP}(165)$ decreased significantly after surgery. That is, the trabeculectomy induced a steepening and a clockwise torsion along the surgical meridian. In bivariate analysis, the combined mean polar values for SIA differed significantly from zero (Hotelling $T^{2}=22.472, p<0.001$ ), which indicates that trabeculectomy was not astigmatically neutral. By the conventional notation, the mean SIA was $0.82 @ 104^{\circ}$. Individual pairs of polar values and the $95 \%$ tolerance ellipse for SIA are described in Fig. 1. Both a net increase in $\mathrm{KP}(120)$ and a net decrease in $\mathrm{KP}(165)$ occurred in 29 eyes $(56.9 \%)$ after surgery.

Table 3 shows the clinical factors affecting the SIA after trabeculectomy. To identify factors having a significant independent effect, MANOVA was performed with only factors of association that trended toward significance (unadjusted $p$ value $<0.1$ ) included as independent variables. The MANOVA (both unadjusted and adjusted for age and follow-up duration) revealed that the surgical site (adjusted $p=0.03$ ) and postoperative IOP (adjusted $p=$ 0.002) had significant effects on the SIA. Together, these factors explained $38.9 \%$ of the total variance in SIA (14.1\% for the surgical site and $24.8 \%$ for the postoperative IOP). The individual vectors of SIA are presented in Fig. 2. We found differences in the distribution of SIA vectors according to the surgical site and postoperative IOP, using a median split at $10 \mathrm{mmHg}$. The mean SIA values were $0.71 @ 114^{\circ}$ in the superotemporal group (25 eyes), $1.00 @ 97^{\circ}$ in the superonasal group (26 eyes), $1.22 @ 102^{\circ}$ in the lower postoperative IOP group (29 eyes), and $0.33 @ 115^{\circ}$ in the higher postoperative IOP group (22 eyes). Meanwhile, there were seven patients who showed the magnitude of SIA greater than 2 diopters. Despite of small number of patients, their mean age was younger (35.1 \pm 14.9 years, $p=0.03$ by Mann-Whitney $U$-test) and their mean postoperative IOP was lower $(7.3 \pm 2.1 \mathrm{mmHg}, p=$ 0.01 by Mann-Whitney $U$-test) than those of patients who showed the magnitude of SIA less than 2 diopters (mean 
age of $50.1 \pm 16.8$ years and mean postoperative IOP of $12.0 \pm 5.9 \mathrm{mmHg})$.

\section{Discussion}

The present study found that SIA changes (steepening and clockwise torsion along the wound) were significant after

Table 3 Effect of clinical factors on surgically induced astigmatism after trabeculectomy

\begin{tabular}{lll}
\hline Factors & $\begin{array}{l}\text { Unadjusted } p \\
\text { value }^{\mathrm{a}}\end{array}$ & $\begin{array}{l}\text { Adjusted } p \\
\text { value }^{\mathrm{b}}\end{array}$ \\
\hline $\begin{array}{l}\text { Age (years) } \\
\text { Site (superotemporal/ }\end{array}$ & 0.09 & 0.19 \\
superonasal) & 0.03 & 0.03 \\
$\begin{array}{l}\text { Axial length (mm) } \\
\text { Follow-up period (months) }\end{array}$ & 0.91 & \\
Preoperative IOP (mmHg) & 0.04 & 0.21 \\
Postoperative IOP (mmHg) & $<0.001$ & \\
$\begin{array}{l}\text { Number of scleral sutures } \\
\text { Number requiring laser }\end{array}$ & 0.58 & 0.002 \\
suture lysis & 0.62 & \\
Number requiring needling & 0.20 & \\
\hline
\end{tabular}

$I O P$ intraocular pressure

${ }^{\text {a }}$ Multivariate analysis of variance with each factor entered as the one independent variable

${ }^{\mathrm{b}}$ Multivariate analysis of variance with factors of unadjusted $p$ value $<0.1$ included as independent variables trabeculectomy, using Naeser's polar value analysis. The mean SIA was greater in eyes with lower postoperative IOP. Moreover, the mean SIA was greater when the surgery was performed on a superonasal quadrant rather than on a superotemporal quadrant. To perform calculations of SIA, net astigmatism must be converted to dioptric vectors. An astigmatism is expressed as direction and magnitude. For statistical analysis, these incommensurable entities must be converted to polar values or similar entities [7]. Naeser described the polar value method with a simplified mathematics based on the sine-squared correlation [11]. Using Naeser's method, the 2 polar values may be analyzed simultaneously with bivariate statistical methods, which is superior to the univariate approach and yields an illustrative graphical display [7]. In addition, by choosing the direction of the surgical plane for $\Phi$ in $\operatorname{KP}(\Phi)$, polar value analysis may be applied on any type of refractive surgery to evaluate the corneal flattening effect and net torsion of the incision [8].

Several studies have focused on SIA after trabeculectomy. Although the results from earlier studies vary in terms of the number of patients, follow-up duration, and surgical techniques used, most report a significant with-therule astigmatism postoperatively [12-16]. Kook et al. investigated corneal astigmatic changes after mitomycin-C augmented trabeculectomy, using the Cravy method, and found that a mean induced astigmatism of $+1.23 @ 90^{\circ}$ at 3 months postoperatively decreased to $+0.65 @ 90^{\circ}$ at 12 months [16]. We found that the magnitude of SIA was comparable $\left(0.82 @ 104^{\circ}\right)$ to those reported in the former
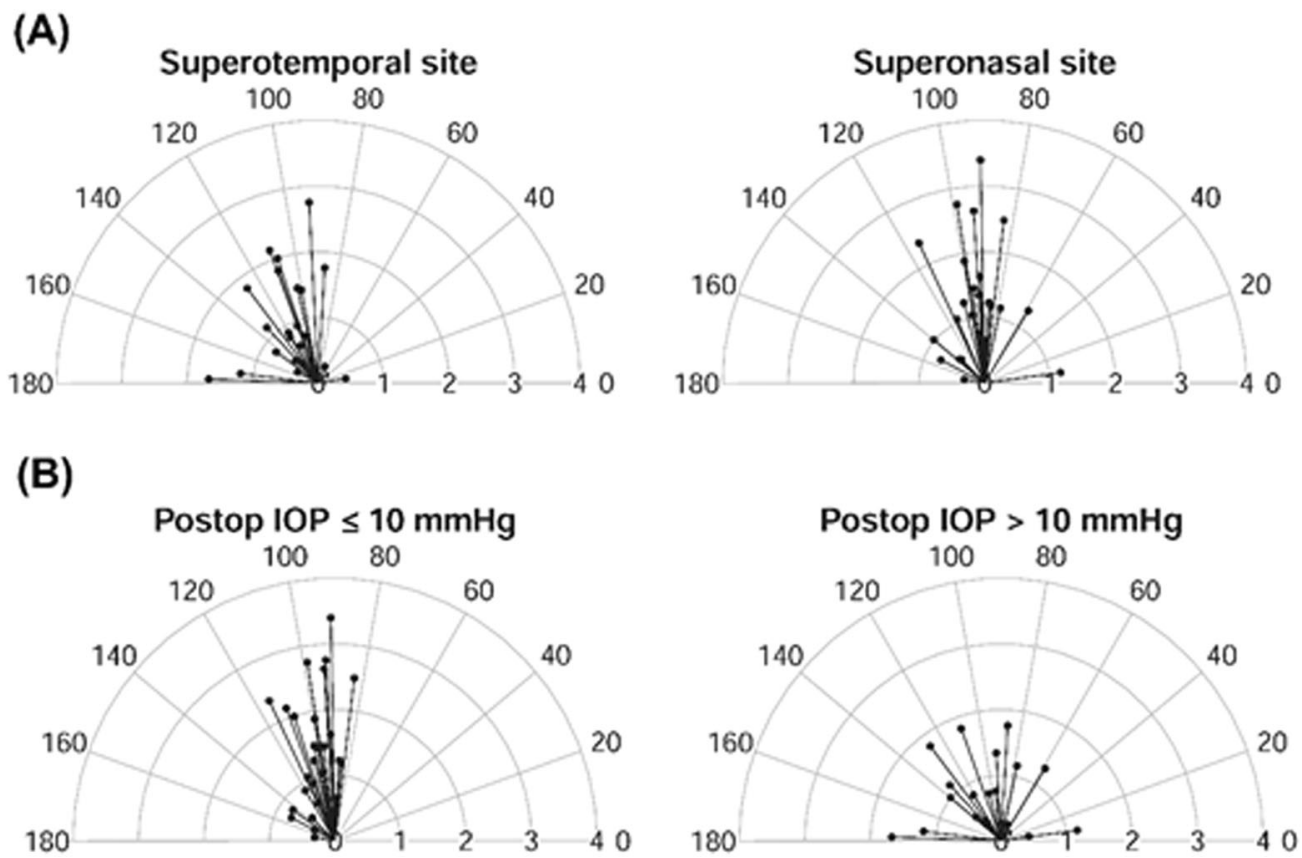

Fig. 2 Individual vectorial display of surgically induced astigmatism according to site (a) and postoperative intraocular pressure (b). IOP intraocular pressure 
studies, although the time of postoperative keratometry measurement was not equal, due to the retrospective nature of the study. When considering the earlier studies defined negligible corneal astigmatism as $\leq 0.5$ diopters of difference between the two principal meridians, the magnitude of mean SIA (0.82 diopters) in this study could be presumed clinically significant in the postoperative visual recovery $[17,18]$.

Factors affecting corneal astigmatism after trabeculectomy have been less investigated than those after cataract surgery. However, Claridge et al. suggested that tissue contraction due to the extensive scleral cauterization, a large drainage bleb, and postoperative ptosis might contribute to corneal astigmatic change [15]. In this study, postoperative IOP and the surgical site (whether it was performed on a superotemporal or superonasal quadrant) showed independent effects on the SIA. A correlation between a lower postoperative IOP and a greater with-the-rule change has been demonstrated to be significant after 1 month, but to disappear after 6 months, by other investigators [12]. This association could be attributed to increased susceptibility to deformation in eyes with lower IOP. An interesting finding in the present study was that trabeculectomy on the superonasal quadrant led to greater SIA than that on the superotemporal quadrant. A similar situation has been reported in the literature for post-cataract surgery [1, 19]. A possible explanation is that the optical center of the cornea is located more nasally and inferiorly to the geometric center and therefore, a superonasal limbus is closer to the optical center than a superotemporal limbus, and has a greater effect on corneal curvature changes. Furthermore, a steeper angle of approach over the nose to a superonasal meridian in the left eye may lead to increased wound stress and higher SIA [20]. On the other hand, the effect of laser suture lysis on SIA was insignificant, consistent with the findings described in previous studies $[12,21]$. And we discovered neither the number of scleral sutures nor needling influenced the SIA. Hugkulstone et al. had also showed that there was no difference in with-the-rule change after trabeculectomy between scleral flaps sutured with 2 or 5 stitches despite the limitation of small number of patients and short follow-up [13].

This study has several limitations. First, because of the retrospective nature of the study, we were unable to perform follow-up examination regularly and observe the changes of SIA over time. However, we attempted to overcome such shortcomings by confining the time of postoperative keratometry measurement to between 2 months and 1 year after surgery, and thus evaluated the SIA in the mid to late postoperative period. Second, as all surgeries were performed by a single experienced surgeon, one should take into account the surgeon factor when applying the outcomes. Nevertheless, the similar outcomes in terms of the pattern and magnitude of SIA in this and earlier studies lend credence to the findings of this study. Third, postoperative care, such as needling and suture lysis, could not be controlled, in that the surgeon determined the need for these interventions to order to achieve the target IOP on each occasion. Fourth, study population was relatively young to be diagnosed with open angle glaucoma and undergo trabeculectomy, which might limit the generalization of the results. As previous studies have shown, the sclera becomes more rigid and noncompliant with age [22]. Therefore, the SIA after trabeculectomy in younger patients might be greater and more affected by factors such as postoperative IOP or surgical site in present study.

In summary, this study provides clinical data on the midto long-term changes in corneal astigmatism after trabeculectomy using vector analysis. SIA appears to be greater in eyes with low postoperative IOP and using a superonasal surgical approach. Based on our results, clinicians may consider choosing a superotemporal rather than a superonasal wound for primary trabeculectomy, particularly in eyes in which the preoperative corneal astigmatism is negligible. Moreover, further studies are needed to identify whether bleb configuration has any effect on the corneal astigmatic change after trabeculectomy.

\section{Summary}

\section{What was known before}

- Trabeculectomy is a common surgical procedure for glaucoma patients, but may induce a certain degree of corneal astigmatic change that impairs visual outcomes.

\section{What this study adds}

- Trabeculectomy along the oblique meridian resulted in significant steepening and clockwise torque along the surgical meridian.

- With-the-rule change after trabeculectomy appears to be greater in eyes with low postoperative IOP and using a superonasal rather than a superotemporal surgical approach.

Acknowledgements We would like to thank all patients who contributed their time and thus allowed us to obtain the data for this publication.

\section{Compliance with ethical standards}

Conflict of interest The authors declare that they have no conflict of interest. 


\section{References}

1. Altan-Yaycioglu R, Akova YA, Akca S, Gur S, Oktem C. Effect on astigmatism of the location of clear corneal incision in phacoemulsification of cataract. J Refract Surg. 2007;23:515-8.

2. Hashemi H, Khabazkhoob M, Soroush S, Shariati R, Miraftab M, Yekta A. The location of incision in cataract surgery and its impact on induced astigmatism. Curr Opin Ophthalmol. 2016;27:58-64.

3. Luo L, Lin H, He M, Congdon N, Yang Y, Liu Y. Clinical evaluation of three incision size-dependent phacoemulsification systems. Am J Ophthalmol. 2012;153:831-9.e2.

4. Denoyer A, Ricaud X, Van Went C, Labbe A, Baudouin C. Influence of corneal biomechanical properties on surgically induced astigmatism in cataract surgery. J Cataract Refract Surg. 2013;39:1204-10.

5. Pfleger T, Skorpik C, Menapace R, Scholz U, Weghaupt H, Zehetmayer M. Long-term course of induced astigmatism after clear corneal incision cataract surgery. J Cataract Refract Surg. 1996;22:72-77.

6. Reddy B, Raj A, Singh VP. Site of incision and corneal astigmatism in conventional SICS versus phacoemulsification. Ann Ophthalmol (Skokie). 2007;39:209-16.

7. Naeser K, Hjortdal JO. Bivariate analysis of surgically induced regular astigmatism. Mathematical analysis and graphical display. Ophthalmic Physiol Opt. 1999;19:50-61.

8. Naeser K. Assessment and statistics of surgically induced astigmatism. Acta Ophthalmol. 2008;86(Suppl 1):5-28.

9. Naeser K, Knudsen EB, Hansen MK. Bivariate polar value analysis of surgically induced astigmatism. J Refract Surg. 2002;18:72-8.

10. Naeser K, Hjortdal J. Polar value analysis of refractive data. J Cataract Refract Surg. 2001;27:86-94.
11. Næser K. A new method to describe the surgically induced change in corneal astigmatism. Acta Ophthalmol. 1990;68(S195):33-6.

12. Delbeke H, Stalmans I, Vandewalle E, Zeyen T. The effect of trabeculectomy on astigmatism. J Glaucoma. 2016;25:e308-12.

13. Hugkulstone $\mathrm{CE}$. Changes in keratometry following trabeculectomy. Br J Ophthalmol. 1991;75:217-8.

14. Cunliffe IA, Dapling RB, West J, Longstaff S. A prospective study examining the changes in factors that affect visual acuity following trabeculectomy. Eye (Lond). 1992;6(Pt 6):618-22.

15. Claridge KG, Galbraith JK, Karmel V, Bates AK. The effect of trabeculectomy on refraction, keratometry and corneal topography. Eye (Lond). 1995;9(Pt 3):292-8.

16. Kook MS, Kim HB, Lee SU. Short-term effect of mitomycin-C augmented trabeculectomy on axial length and corneal astigmatism. J Cataract Refract Surg. 2001;27:518-23.

17. Tejedor J, Perez-Rodriguez JA. Astigmatic change induced by 2.8-mm corneal incisions for cataract surgery. Invest Ophthalmol Vis Sci. 2009;50:989-94.

18. Tejedor J, Murube J. Choosing the location of corneal incision based on preexisting astigmatism in phacoemulsification. Am J Ophthalmol. 2005;139:767-76.

19. Ozkurt Y, Erdogan G, Guveli AK, Oral Y, Ozbas M, Comez AT, et al. Astigmatism after superonasal and superotemporal clear corneal incisions in phacoemulsification. Int Ophthalmol. 2008;28:329-32.

20. Kohnen S, Neuber R, Kohnen T. Effect of temporal and nasal unsutured limbal tunnel incisions on induced astigmatism after phacoemulsification. J Cataract Refract Surg. 2002;28:821-5.

21. Rosen WJ, Mannis MJ, Brandt JD. The effect of trabeculectomy on corneal topography. Ophthalmic Surg. 1992;23:395-8.

22. Pallikaris IG, Kymionis GD, Ginis HS, Kounis GA, Tsilimbaris MK. Ocular rigidity in living human eyes. Invest Ophthalmol Vis Sci. 2005;46:409-14. 